\title{
Analysis and optimisation for inerter-based isolators via fixed-point theory and algebraic solution
}

\author{
Yinlong $\mathrm{Hu}^{\mathrm{a}}$, Michael Z. Q. Chen ${ }^{\mathrm{b}, \mathrm{c}, *}$, Zhan Shu ${ }^{\mathrm{d}}$, Lixi Huang ${ }^{\mathrm{b}, \mathrm{e}}$ \\ ${ }^{a}$ School of Automation, Nanjing University of Science and Technology, Nanjing, China \\ ${ }^{b}$ Department of Mechanical Engineering, The University of Hong Kong, Hong Kong. \\ ${ }^{c}$ Shenzhen Institute of Research and Innovation, The University of Hong Kong, Shenzhen, China. \\ ${ }^{d}$ Faculty of Engineering and the Environment, University of Southampton, Southampton, U.K. \\ ${ }^{e}$ Lab for Aerodynamics and Acoustics, Zhejiang Institute of Research and Innovation, The University of \\ Hong Kong, Zhejiang, China.
}

\begin{abstract}
This paper is concerned with the problem of analysis and optimisation of the inerter-based isolators based on a "uni-axial" single-degree-of-freedom isolation system. In the first part, in order to gain an in-depth understanding of inerter from the prospective of vibration, the frequency responses of both parallel-connected and series-connected inerters are analysed. In the second part, three other inerter-based isolators are introduced and the tuning procedures in both the $H_{\infty}$ optimisation and the $H_{2}$ optimisation are proposed in an analytical manner. The achieved $H_{2}$ and $H_{\infty}$ performance of the inerter-based isolators is superior to that achieved by the traditional dynamic vibration absorber (DVA) when the same inertance-tomass (or mass) ratio is considered. Moreover, the inerter-based isolators have two unique properties, which are more attractive than the traditional DVA: first, the inertance-to-mass ratio of the inerter-based isolators can easily be larger than the mass ratio of the traditional DVA without increasing the physical mass of the whole system; second, there is no need to mount an additional mass on the object to be isolated.
\end{abstract}

Keywords: Inerter, vibration isolation, $H_{\infty}$ optimisation, $H_{2}$ optimisation.

\section{Introduction}

Inerter is a two-terminal mechanical device with the property that the applied force at its two terminals is proportional to the relative acceleration between them $[1,2]$, where the constant of proportionality is called inertance with a unit of kilogram. Since the initial application in Formula One racing car suspension systems [2], inerters have been applied to various mechanical systems mainly including vehicle suspensions $[3,4,5,6,7,8,9]$ and vibration suppression $[10,11,12,13,14]$. The interest in passive network synthesis has also been rekindled $[15,16,17,18,19,20,21,22]$. The influence of inerter on vibration systems' natural frequencies has been investigated in [23], where the fundamental property that inerter can reduce natural frequencies of vibration systems has been theoretically demonstrated.

In this paper, to further investigate the influence of inerter on vibration systems, the performance of the inerter-based isolators based on a "uni-axial" single-degree-of-freedom isolation system is studied. First, to gain an in-depth understanding of inerter from the perspective of vibration, the frequency responses of both parallel-connected and series-connected

${ }^{*}$ Corresponding author. Email: mzqchen@hku.hk. 
inerters are analysed. It is shown that an extra invariant point, which is independent of the damping ratio, can be introduced by using the series-connected inerter. Then, to further tune the invariant points, three other inerter-based isolators, each of which incorporates a spring, a damper and an inerter, are proposed. To facilitate the practical application, the optimal parameters of the inerter-based isolators in both $H_{\infty}$ optimisation and $H_{2}$ optimisation are analytically derived. The $H_{\infty}$ optimisation aims to minimise the maximum magnitude of the frequency response based on the fixed-point theory [24] which has been extensively used in tuning the parameters of dynamic vibration absorbers (DVA) (or tuned mass dampers (TMD)) $[25,26,27]$. While the $H_{2}$ optimisation aims to minimise the mean squared displacement of the object under random excitation [29]. An analytical method is employed to calculate the $\mathrm{H}_{2}$ norm performance measures of the inerter-based isolators in this paper. In addition, the comparisons of the $H_{2}$ and $H_{\infty}$ performances between the inerter-based isolators and the traditional DVA show the superiority of the inerter-based isolators. Two properties make the inerter-based isolators potentially more attractive than the traditional DVA: first, a relatively large inertance can easily be obtained without increasing the physical mass of the whole system [1]; second, there is no need to mount an additional mass on the object to be isolated, as an inerter is a built-in component in the inerter-based isolators.

In [12], one of the inerter-based isolators proposed in this paper $(C 3$ in Fig. 7 of this paper) has been employed to reduce vibrations in civil engineering structures, and a $H_{\infty}$ tuning procedure for this configuration has been proposed by using the fixed-point theory [24]. The main difference between the procedures in [12] and the $H_{\infty}$ optimisation proposed in this paper is that the optimal parameters in [12] are obtained through using iterative algorithms while the optimal parameters in this paper are obtained analytically. The analytical method alleviates possible numerical problems induced by iterations and reveals fundamental relationship between tuning parameters and $H_{\infty}$ performance. Detailed difference can be found in Section 4.

The organization of this paper is as follows. In Section 2, a "uni-axial" isolation system is introduced where the force and displacement transmissibilities are also derived. Section 3 provides an in-depth analysis of the frequency response of two simple configurations with inerter to highlight the fundamental properties of inerter in vibration. Section 4 and Section 5 derive the analytical solutions of the inerter-based isolators in $H_{\infty}$ optimisation and $\mathrm{H}_{2}$ optimisation, respectively, where the comparisons with the traditional DVA are also given. Conclusions are drawn in Section 6.

\section{Isolation system description}

In this paper, a "uni-axial" isolation system is considered, as shown in Fig. 1, where the mass $m$ is the object to be isolated, the mass $m_{f}$ is the foundation, and $Q(s)$ is the isolator to be designed. In practice, two situations are commonly encountered depending on the circumstances. One is that the object must be isolated from the objectionable vibratory motions of the supporting surface, while the other is that the supporting surface must be protected from the dynamic load generated within the object. The former situation is called the displacement transmissibility problem and the later one is the force transmissibility problem [31]. In some cases, both tasks have to be addressed simultaneously [30]. For linear isolators, the displacement transmissibility problem and the force transmissibility 
Table 1: $W(s)$ for configurations in Fig. 2 and Fig. 7, where $s$ denotes the Laplace variable.

\begin{tabular}{lll}
$W_{1}(s)=b s+c$ & $W_{2}(s)=\frac{1}{\frac{1}{c}+\frac{1}{b s}}$ & $W_{3}(s)=\frac{1}{\frac{1}{\frac{k_{1}}{s}+c}+\frac{1}{b s}} \quad W_{4}(s)=\frac{1}{\frac{s}{k_{1}}+\frac{1}{b s}+\frac{1}{c}} \quad W_{5}(s)=\frac{1}{\frac{1}{b s+c}+\frac{s}{k_{1}}}$ \\
\hline
\end{tabular}

problem are equivalent if the mass of the foundation is sufficiently larger than that of the object [31]. For brevity, in this paper, the assumption that $m_{f}=\infty$ is made and the absolute displacement transmissibility and the absolute force transmissibility are identically treated as

$$
\mu=\frac{\left|F_{i}\right|}{|F|}=\frac{\left|x_{1}\right|}{\left|x_{2}\right|}=\frac{|Q(\mathrm{j} \omega) \mathrm{j} \omega|}{\left|Q(\mathrm{j} \omega) \mathrm{j} \omega-m \omega^{2}\right|},
$$

where $F$ is the force imposed on the object $m, F_{i}$ is the force generated by the isolator, $x_{1}$ and $x_{2}$ are the displacements of the object and the foundation, respectively. $Q(\mathrm{j} \omega)$ is obtained by replacing the Laplace variable $s$ in $Q(s)$ with $\mathrm{j} \omega$, where $\mathrm{j}$ is a complex variable with $\mathrm{j}^{2}=-1$ and $Q(s)$ is the admittance of the isolator, i.e. the ratio of the applied force $F_{i}$ over the relative velocity $\dot{x}_{1}-\dot{x}_{2}$ in Laplace domain.

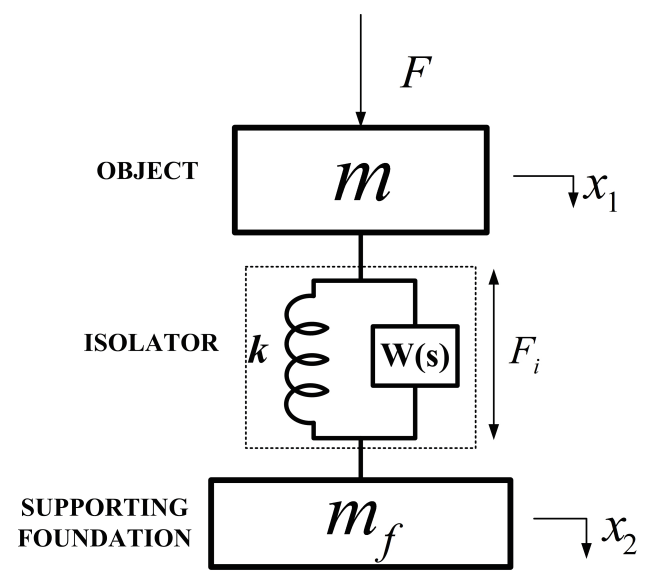

Figure 1: Uni-axial vibration isolation system.

As shown in Fig. 1, $Q(s)=\frac{k}{s}+W(s)$, where $W(s)$ denotes the admittances of passive networks consisting of finite inter-connections of springs, dampers and inerters. In this paper, five inerter-based isolators will be investigated, as shown in Fig. 2 and Fig. 7. Their admittances are summarized in Table 1.

To obtain a dimensionless representation, $\omega_{n}=\sqrt{\frac{k}{m}}$ and $c_{r}=2 \omega_{n} m=2 \sqrt{m k}$ are used to denote the natural frequency and the critical damping of the isolation system shown in Fig. 1 without $W(s)$, respectively. Also, $q=\frac{\omega}{\omega_{n}}, \zeta=\frac{c}{c_{r}}, \delta=\frac{b}{m}$ and $\lambda=\frac{k}{k_{1}}$ denote the frequency ratio, the damping ratio, the inertance-to-mass ratio, and the stiffness ratio, respectively.

For the considered configurations as shown in Fig. 2 and Fig. 7, the transmissibility $\mu$ can be obtained by substituting $Q_{i}(\mathrm{j} \omega)=\frac{k}{\mathrm{j} \omega}+W_{i}(\mathrm{j} \omega), i=1, \ldots, 5$, into $(1)$, respectively, where $W_{i}(\mathrm{j} \omega)$ are given in Table 1 by replacing $s$ with $\mathrm{j} \omega$. 


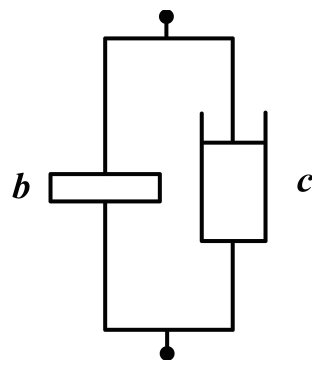

(a)

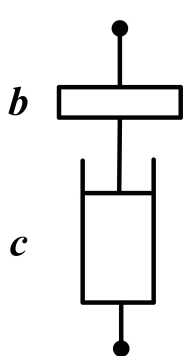

(b)

Figure 2: Two simple configurations as $W(s)$ of the isolators in Fig. 1. (a) C1; (b) C2.

\section{Vibration analysis for two simple inerter-based isolators}

This section is to analyse the fundamental properties of inerter from the perspective of vibration. Note that among all the applications of inerter, the main focus is to optimise some inerter-based mechanical networks possessing more complex structures than the conventional networks consisting of only springs and dampers. The proposed mechanical networks can be obtained either by using networks synthesis $[8,9,22]$ or by giving some fixed-structure networks $[3,4,5,6,7,10,12,14]$. Although the benefits of using inerter can be effectively demonstrated by these complex inerter-based mechanical networks, some fundamental properties of inerter in vibration are overlooked due to the complexity of the structure. Consequently, it lacks in-depth understanding of inerter from the perspective of vibration. In [23], the property that inerter can reduce vibration systems' natural frequencies is demonstrated. However, the influences of inerter on other aspects such as the invariant property in frequency domain are still unclear. This motivated the investigation of this section based on two simple inerter-based configurations, as shown in Fig. 2. The detailed analysis of the frequency responses of these configurations constitutes the main contribution of this section.

\subsection{Analysis of $C 1$}

For this configuration, the transmissibility can be obtained as

$$
\mu=\frac{\left|k-b \omega^{2}+\mathrm{j} c \omega\right|}{\left|k-(m+b) \omega^{2}+\mathrm{j} c \omega\right|}=\sqrt{\frac{\left(1-\delta q^{2}\right)^{2}+(2 \zeta q)^{2}}{\left(1-(1+\delta) q^{2}\right)^{2}+(2 \zeta q)^{2}}} .
$$

Fig. 3 shows the transmissibility $\mu$ with respect to different $\delta$ and $\zeta$, where it is shown that an anti-resonant frequency (a particular frequency where minimum magnitude is obtained) and an invariant point (a particular frequency where the magnitude is independent of the damping ratio $\zeta$ ) are introduced by using the parallel-connected inerter. For the undamped case, the anti-resonant frequency $q_{b}$ can be obtained as $q_{b}=\sqrt{\frac{1}{\delta}}$, and the resonant frequency or natural frequency is $q_{p}=\sqrt{\frac{1}{1+\delta}}$. Note that the natural frequency $q_{p}$ is a decreasing function with respect to $\delta$, which is consistent with the result in [23].

The transmissibility $\mu$ in (2) can be rewritten as

$$
\mu=\sqrt{\frac{A \zeta^{2}+B}{C \zeta^{2}+D}}
$$


116

where $A=4 q^{2}, B=\left(1-\delta q^{2}\right)^{2}, C=4 q^{2}$, and $D=\left(1-(1+\delta) q^{2}\right)^{2}$. To find the invariant points which are independent of damping, it requires

$$
\frac{A}{C}=\frac{B}{D}
$$

that is,

$$
\frac{\left(1-\delta q^{2}\right)^{2}}{\left(1-(1+\delta) q^{2}\right)^{2}}=1
$$

Then, one obtains the nonzero invariant point $q_{i}$ as

$$
q_{i}=\sqrt{\frac{2}{1+2 \delta}}
$$

Obviously, $q_{i}$ is a decreasing function with respect to $\delta$, which means that the parallelconnected inerter can effectively shift the invariant point left.

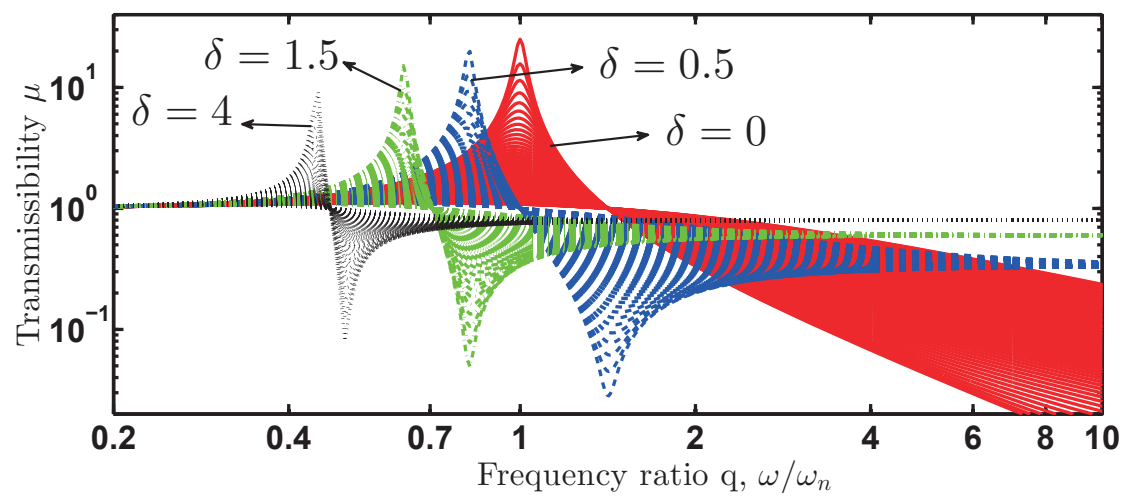

Figure 3: Transmissibility $\mu$ for the configuration $C 1$ when $\zeta$ ranges from 0.02 to 1.2 .

Fig. 4 depicts the transmissibility $\mu$ of configuration $C 1$ when $\delta=1$ with some typical $\zeta$. The magnitudes at the natural frequency $q_{p}$, the anti-resonant frequency $q_{b}$, and infinity can

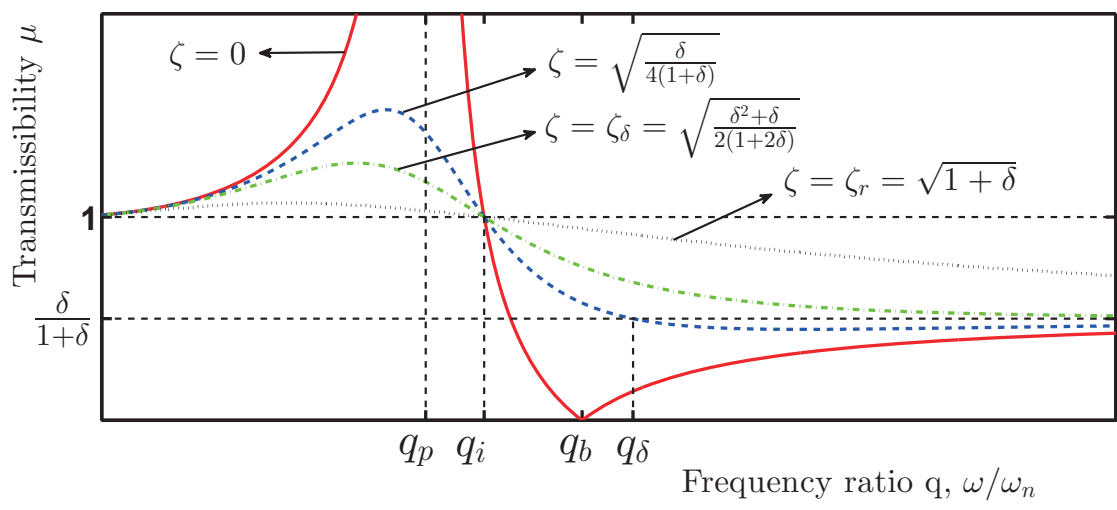

Figure 4: Transmissibility $\mu$ for the configuration $C 1$ when $\delta=1$. 
be obtained as:

$$
\begin{aligned}
\left.\mu\right|_{q=q_{p}} & =\frac{1}{2} \sqrt{\frac{1}{\zeta^{2}(1+\delta)}+4}, \\
\left.\mu\right|_{q=q_{b}} & =2 \sqrt{\frac{1}{\frac{1}{\zeta^{2} \delta}+4}}, \\
\left.\mu\right|_{q \rightarrow \infty} & =\frac{\delta}{1+\delta},
\end{aligned}
$$

where $\left.\mu\right|_{q=q_{j}}$ means the value of $\mu$ when $q=q_{j}, j$ denotes $p, b$ or $\infty$.

From (3) and (4), it is clear that $\left.\mu\right|_{q=q_{p}}$ is a decreasing function with respect to both $\delta$ and $\zeta$, and $\left.\mu\right|_{q=q_{b}}$ is an increasing function with respect to both $\delta$ and $\zeta$, as shown in Fig. 3. From (4), one obtains that for the undamped case, i.e., $c=0$ or $\zeta=0,\left.\mu\right|_{q=q_{b}}=0$, the effect of "dynamic absorption" of vibration occurs, which is uncommon for single-degree-of-freedom systems [30].

Equation (5) shows that the transmissibility approaches to an asymptote at the level of $\frac{\delta}{1+\delta}$ when $q$ tends to $\infty$. For a given $\delta$, by solving the equation

$$
\mu=\sqrt{\frac{\left(1-\delta q^{2}\right)^{2}+(2 \zeta q)^{2}}{\left(1-(1+\delta) q^{2}\right)^{2}+(2 \zeta q)^{2}}}=\frac{\delta}{1+\delta}
$$

one obtains that

$$
q_{\delta}=\frac{\sqrt{2}}{2} \sqrt{\frac{1+2 \delta}{\delta^{2}+\delta-2 \zeta^{2}(1+2 \delta)}} .
$$

Note that $q_{\delta}$ is real if and only if $\zeta<\zeta_{\delta}=\sqrt{\frac{\delta^{2}+\delta}{2(1+2 \delta)}}$. Since the transmissibility tends to an asymptote at the level of $\frac{\delta}{1+\delta}$ when $q$ tends to $\infty, \zeta_{\delta}$ is a critical value of $\zeta$ in the sense that: if $\zeta<\zeta_{\delta}$, there exists a finite $q$ where the minimum of $\mu$ occurs; otherwise, $\mu$ is uniformly larger than $\frac{\delta}{1+\delta}$ and approaches $\frac{\delta}{1+\delta}$ when $q$ tends to $\infty$. The curve with $\zeta=\zeta_{\delta}$ is shown in Fig. 4.

Note that $q_{p}$ and $q_{b}$ are the natural frequency and the anti-resonant frequency of the undamped case, respectively. For the damped case, the real natural frequency $q_{p r}$ and antiresonant frequency $q_{b r}$ for a specific damping ratio $\zeta$, can be obtained by setting the derivative of (2) to zero. Then, one obtains

$$
\begin{aligned}
& q_{p r}=\sqrt{\frac{1+2 \delta-\sqrt{1+8 \zeta^{2}(1+2 \delta)}}{2\left(\delta^{2}+\delta-2 \zeta^{2}(1+2 \delta)\right)}}, \\
& q_{b r}=\sqrt{\frac{1+2 \delta+\sqrt{1+8 \zeta^{2}(1+2 \delta)}}{2\left(\delta^{2}+\delta-2 \zeta^{2}(1+2 \delta)\right)}} .
\end{aligned}
$$

It is clear that if $\zeta \approx 0, q_{p r} \approx q_{p}$ and $q_{b r} \approx q_{b}$ hold, but for a large $\zeta$, it is not sufficient to use this estimation.

In summary, one obtains the following remarks. 
Remark 1. 1. The parallel-connected inerter can effectively lower the invariant point that independent of the damping ratio $\zeta$;

2. The magnitude at the natural frequency is a decreasing function with respect to both the damping ratio and the inertance-to-mass ratio; the magnitude at the anti-resonant frequency is an increasing function with respect to both the damping ratio and the inertance-to-mass ratio;

3. The isolation at high frequencies is weakened by using the parallel-connected inerter, where the magnitude tends to $\frac{\delta}{1+\delta}$ when $q$ tends to $\infty$.

\subsection{Analysis of $C^{2} 2$}

For this configuration, the transmissibility can be obtained as

$$
\begin{aligned}
\mu & =\frac{\left|\frac{k c}{b}-c \omega^{2}+k j \omega\right|}{\left|\frac{k c}{b}-c \omega^{2}-\frac{m c}{b} \omega^{2}+\left(k-m \omega^{2}\right) j \omega\right|}, \\
& =\sqrt{\frac{\delta^{2} q^{2}+4\left(1-\delta q^{2}\right)^{2} \zeta^{2}}{\delta^{2}\left(1-q^{2}\right)^{2} q^{2}+4\left(1-(1+\delta) q^{2}\right)^{2} \zeta^{2}}} .
\end{aligned}
$$

By rewriting (10) as

$$
\mu=\sqrt{\frac{A \zeta^{2}+B}{C \zeta^{2}+D}},
$$$$
\text { (1) }
$$

$$
2 \text { in }
$$

the magnitudes at $P$ and $Q$ are

$$
\left.\mu\right|_{q=q_{P}}=\left|\frac{1}{1-q_{P}^{2}}\right|,\left.\mu\right|_{q=q_{Q}}=\left|\frac{1}{1-q_{Q}^{2}}\right| .
$$




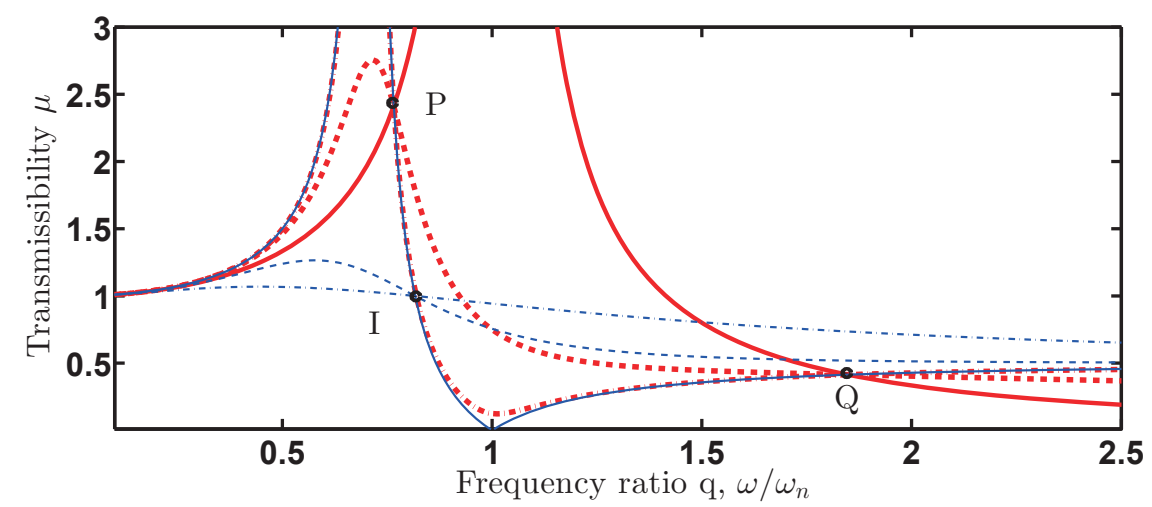

Figure 5: Comparison of the transmissibilities of configurations $C 1$ and $C 2$ when $\delta=1$. Red bold lines denote $C 2$ and blue thin lines denote $C 1$. The solid lines denote $\zeta=0$; the dash lines denote $\zeta=\zeta_{\delta}=0.5774$; the dash-dot lines denote $\zeta=\zeta_{r}=\sqrt{1+\delta}=\sqrt{2}$.

Since $q_{P}^{2}<1$ and $q_{Q}^{2}>2$, one obtains

$$
\left.\mu\right|_{q=q_{P}}>1>\left.\mu\right|_{q=q_{Q}},
$$

which means that for a finite $\delta$, it is impossible to equalise the ordinates at the two invariant points.

A comparison of the transmissibilities of configurations $C 1$ and $C 2$ is shown in Fig. 5, where two invariant points $P$ and $Q$ of configuration $C 2$ are depicted. It is shown that for the same damping ratio $\zeta$, the behaviors of configurations $C 1$ and $C 2$ are totally different. For example, for the case of $\zeta=\zeta_{r}=\sqrt{2}$ (dash-dot lines in Fig. 5), $C 1$ is overdamped while $C 2$ behaves similarly to the undamped case of $C 1$. This is caused by the series structure of $C 2$, as by varying the damping ratio $\zeta$ from 0 to $\infty$, the configuration $C 2$ is changed from the configuration with only a spring to the configuration with a parallel connection of a spring and an inerter.

In summary, one obtains the following remarks.

Remark 2. 1. Two invariant points, which are independent of the damping ratio, can be introduced by using the series-connected inerter, and both the two invariant points are decreasing functions with respect to the inertance-to-mass ratio;

2. For a finite inertance-to-mass ratio, the magnitude at the smaller invariant point is larger than 1 and the magnitude at the larger invariant point is smaller than 1;

3. The series arrangement $C 2$ behaves between the configuration with only a spring and the configuration with a parallel connection of a spring and an inerter.

\section{4. $H_{\infty}$ optimisation for inerter-based isolators}

In practice, in order to achieve good isolating performance, it is always desirable to minimise the maximum displacement of the object, which is known as $H_{\infty}$ optimisation [26]. In the previous section, it is shown that the invariant point, the resonant frequency and the anti-resonant frequency are directly determined by the inertance-to-mass ratio $\delta$. Therefore, in this section, $H_{\infty}$ tuning procedures for a given $\delta$ will be proposed. 


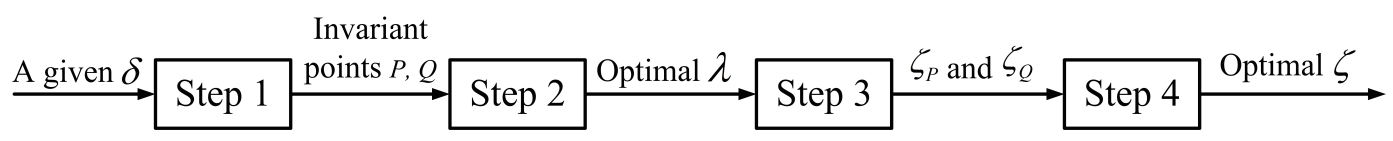

Figure 6: Graphical representation of Procedure 1.

For the configuration $C 1$ in Fig. 2, the optimal damping in $H_{\infty}$ optimisation for a given $\delta$ is $\infty$, which is a trivial solution, as in this case the object and the foundation are stiffly connected. For the configuration $C 2$ with a given inertance-to-mass ratio $\delta$, the optimal damping ratio $\zeta$ for the $H_{\infty}$ performance is the one making the curve horizontally pass though the invariant $P$, as shown in Fig. 5. The rationality is based on the fixed-point theory [24, Chapter 3.3]: the most favorable damping is the one making the curve horizontally pass through the highest invariant point. As demonstrated in Section 3, the magnitude of the invariant point $P$ is always larger than that of the other invariant point $Q$. Therefore, based on this consideration, the optimal damping ratio $\zeta$ for configuration $C 2$ can be obtained as follows:

Proposition 1. For the configuration $C 2$ with a given $\delta$, the optimal damping ratio $\zeta$ in $H_{\infty}$ optimisation is

$$
\zeta_{\text {opt }}=\frac{1}{2} \sqrt{\delta\left(1+\delta-\sqrt{1+\delta^{2}}\right)} .
$$

Proof. See Appendix Appendix A.

Note that two invariant points can be introduced by using the series-connected inerter, and in order to further tune the two invariant points, an extra spring $k_{1}$ is incorporated. Then, three inerter-based isolators are proposed as shown in Fig. 7. The fixed-point theory [24, Chapter 3.3] is employed to derive the optimal parameters for these three inerter-based isolators. The fixed-point theory can be summarised as follows [24, Chapter 3.3].

Procedure 1. 1. For a given inertance-to-mass ratio $\delta$, find the invariant points which are independent of the damping ratio $\zeta$, and denote the two smaller invariant points as $P$ and $Q$;

2. adjust the spring stiffness ratio $\lambda$ so that the ordinates at the invariant points $P$ and $Q$ are equal;

3. calculate the damping ratio $\zeta_{P}$ and $\zeta_{Q}$ so that the curves of transmissibility $\mu$ vs. $q$ horizontally pass through $P$ and $Q$, respectively;

4. obtain the optimal damping ratio as $\zeta=\sqrt{\frac{\zeta_{P}^{2}+\zeta_{Q}^{2}}{2}}$.

A graphical representation of Procedure 1 is given in Fig. 6, indicating the required and output parameters in each step. According to this procedure, the optimal parameters $\lambda$ and $\zeta$ for each configuration are derived subsequently.

Remark 3. The fixed-point theory [24, Chapter 3.3] actually yields a suboptimal but highly precise solution as demonstrated in [33]. The merit of the fixed-point theory is that an analytical solution can be easily derived, which makes it extensively employed in tuning dynamic vibration absorber (DVA) (or tuned mass damper (TMD)). See for example [25, 26, 27] and 


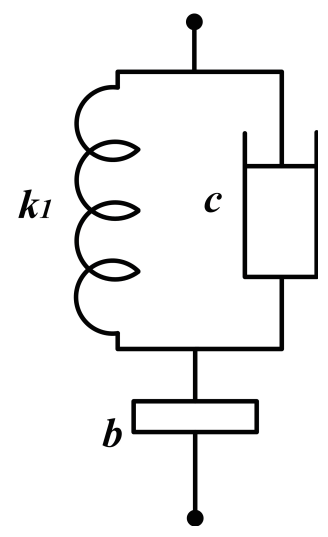

(a)

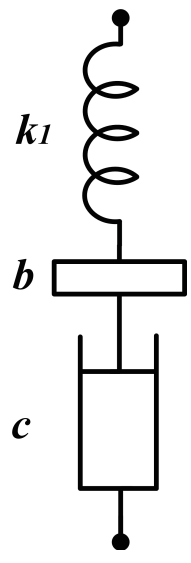

(b)

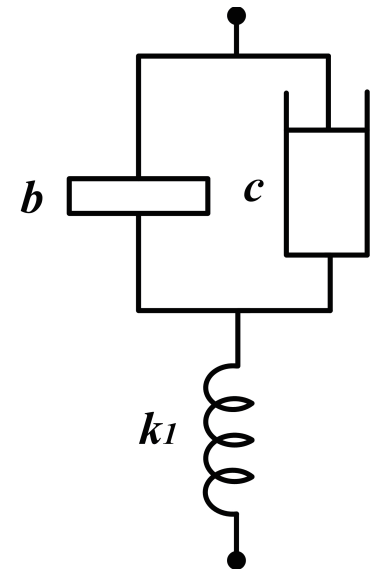

(c)

Figure 7: Three configurations as $W(s)$ of the isolators in Fig. 1. (a) C3; (b) C4; (c) C5.

$$
\zeta_{P, Q}^{2}=\left(\frac{1-\delta(1+\lambda) q_{P, Q}^{2}}{1-\delta q_{P, Q}^{2}}\right)\left(\frac{\delta(1+\lambda)\left(2-(1+2 \delta) q_{P, Q}^{2}\right)-\left(2 \delta \lambda q_{P, Q}^{2}-1\right)\left(1-\delta q_{P, Q}^{2}\right)}{4 \lambda^{2} q_{P, Q}^{2}}\right),
$$

which possesses a relatively large value $\left(q_{R}^{2} \geq 3\right)$. The optimal stiffness ratio $\lambda$ can be obtained as

$$
\lambda=\frac{2\left(q_{R}^{4} \delta(1+\delta)-(1+2 \delta) q_{R}^{2}+1\right)}{\delta q_{R}^{2}\left(q_{R}^{4} \delta-2(\delta+1) q_{R}^{2}+2\right)} \text { or } \frac{2\left((1+2 \delta)(1+\delta) q_{R}^{2}-2(1+\delta)\right)}{q_{R}^{2}\left(\delta(1+2 \delta) q_{R}^{2}-2\left(1+2 \delta+2 \delta^{2}\right)\right)} .
$$

The optimal damping ratio $\zeta$ can be obtained as

$$
\zeta=\sqrt{\frac{\zeta_{P}^{2}+\zeta_{Q}^{2}}{2}}
$$

where $\zeta_{P}^{2}$ and $\zeta_{Q}^{2}$ can be obtained as 


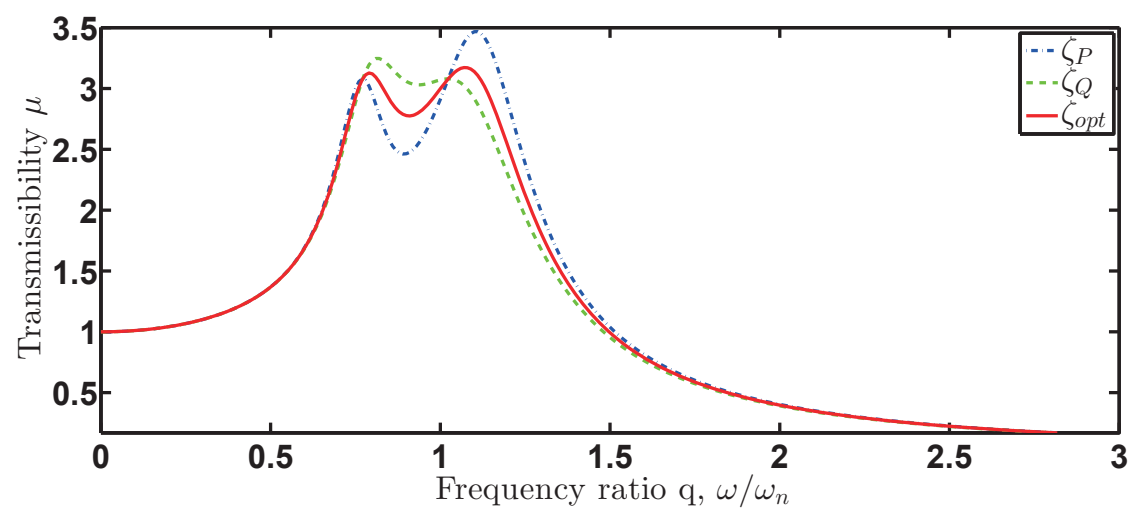

Figure 8: Transmissibility $\mu$ for $C 3$ when $\delta=0.2$.

233

234

$q_{P}^{2}$ and $q_{Q}^{2}$ are solutions of the following quadratic function with respect to $q^{2}$ :

$$
q^{4}-\left(\frac{2}{\delta \lambda}(1+\lambda+\delta+\lambda \delta)-q_{R}^{2}\right) q^{2}+\frac{2}{\delta^{2} \lambda q_{R}^{2}}=0 .
$$

Proof. See Appendix Appendix B.

Procedure 2. In summary, the $H_{\infty}$ tuning procedure for $C 3$ is:

1. obtain $q_{R}$ from (15);

2. obtain $\lambda_{\text {opt }}$ by substituting $q_{R}$ into (16);

3. obtain $q_{P}$ and $q_{Q}$ by solving (19);

4. obtain $\zeta_{p}^{2}$ and $\zeta_{Q}^{2}$ by substituting $q_{P}$ and $q_{Q}$ into (18), respectively;

5. obtain the optimal $\zeta_{\text {opt }}$ from (17).

Note that in [12, Section 3], a similar tuning procedure was given for the configuration $C 3$ by following the procedure given in [24, Chapter 3.3] as well. The main difference between the method in this paper and the one in [12] is the approach in calculating the optimal parameters $\lambda$ and $\zeta$ : In this paper, the analytical solutions of the optimal $\lambda$ and $\zeta$ are given, that is, (15), (16), and (18); while in [12], the optimal $\lambda$ and $\zeta$ are obtained relying on numerical iterations. Hence, the procedure in this paper is more convenient and reliable.

The transmissibility $\mu$ of $C 3$ for $\delta=0.2$ is illustrated in Fig. 8 .

Proposition 3. The transmissibility for $C 4$ can be obtained as

$$
\mu=\left|\frac{2\left(1-\delta(1+\lambda) q^{2}\right) \zeta+\mathrm{j} \delta q}{2\left(\delta \lambda q^{4}-(1+\delta+\delta \lambda) q^{2}+1\right) \zeta+\mathrm{j} \delta\left(1-q^{2}\right) q}\right|
$$

Following Procedure 1, the optimal stiffness ratio $\lambda$ can be obtained as

$$
\lambda=\frac{1}{\delta} .
$$

The optimal damping ratio $\zeta$ can be obtained as

$$
\zeta_{o p t}=\sqrt{\frac{\zeta_{P}^{2}+\zeta_{Q}^{2}}{2}},
$$




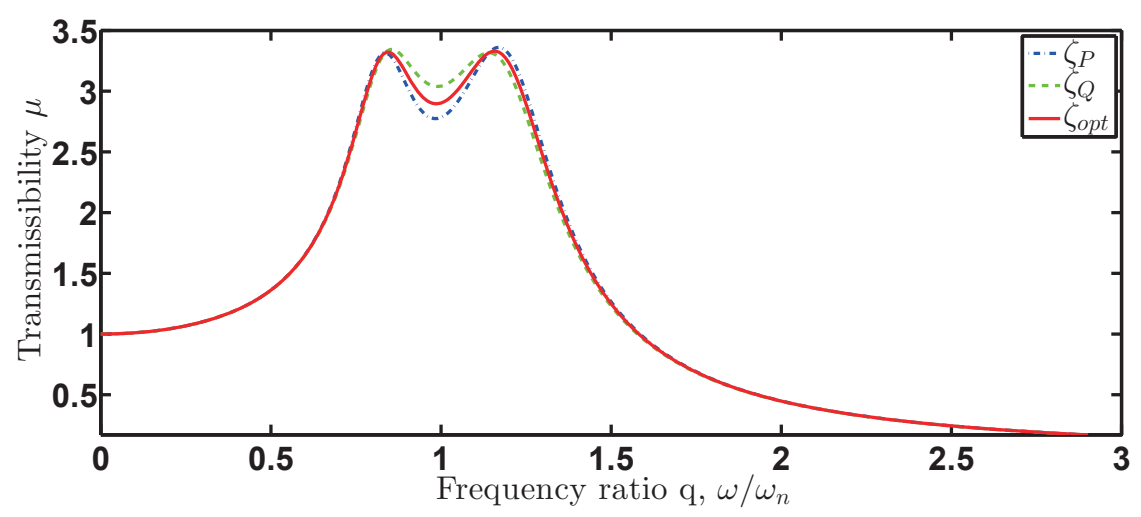

Figure 9: Transmissibility $\mu$ for $C 4$ when $\delta=0.2$.

251

where

$$
\begin{aligned}
\zeta_{P}^{2} & =\frac{\delta^{2}(1-\sqrt{\delta /(2+\delta)})}{4((1+\delta) \sqrt{\delta /(2+\delta)}-\delta)((\delta+3) \sqrt{\delta /(2+\delta)}+\delta)}, \\
\zeta_{Q}^{2} & =\frac{\delta^{2}(1+\sqrt{\delta /(2+\delta)})}{4((1+\delta) \sqrt{\delta /(2+\delta)}+\delta)((\delta+3) \sqrt{\delta /(2+\delta)}-\delta)}
\end{aligned}
$$

Proof. See Appendix Appendix C.

The transmissibility $\mu$ of $C 4$ for $\delta=0.2$ is illustrated in Fig. 9.

Proposition 4. The transmissibility for C5 can be obtained as

$$
\mu=\left|\frac{1-\delta(1+\lambda) q^{2}+\mathrm{j} 2(\lambda+1) \zeta q}{1-(1+\delta+\delta \lambda) q^{2}+\delta \lambda q^{4}+\mathrm{j} 2 \zeta\left(\lambda+1-\lambda q^{2}\right) q}\right| .
$$

Following Procedure 1, the optimal stiffness ratio $\lambda$ can be obtained as

$$
\lambda=\frac{1}{2 \delta}(1-2 \delta+\sqrt{1-2 \delta})
$$

which requires $\delta<1 / 2$. The optimal damping ratio $\zeta$ can be obtained as

$$
\zeta_{o p t}=\sqrt{\frac{\zeta_{P}^{2}+\zeta_{Q}^{2}}{2}}
$$

257 where

$$
\zeta_{P, Q}^{2}=\frac{\left(1-\delta(1+\lambda) q_{P, Q}^{2}\right)\left(1+2 \delta+2 \delta \lambda-3 \delta \lambda q_{P, Q}^{2}\right)}{4(\lambda+1) \lambda q_{P, Q}^{2}}
$$

and

$$
q_{P, Q}^{2}=\frac{1}{4 \delta \lambda(\lambda+1)}\left(1+2 \lambda+2 \delta(1+\lambda)^{2} \pm \sqrt{\left(2 \delta(1+\lambda)^{2}+1-2 \lambda\right)^{2}+8 \lambda}\right) .
$$

Proof. See Appendix Appendix D.

The transmissibility $\mu$ of $C 5$ for $\delta=0.2$ is illustrated in Fig. 10 . 




Figure 10: Transmissibility $\mu$ for $C 5$ when $\delta=0.2$.

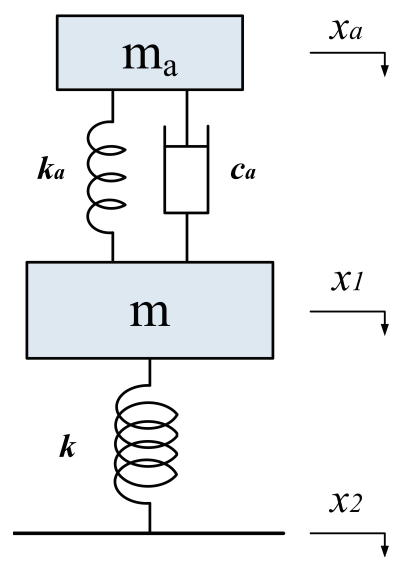

Figure 11: The dynamic vibration absorber attached to the object mass. 


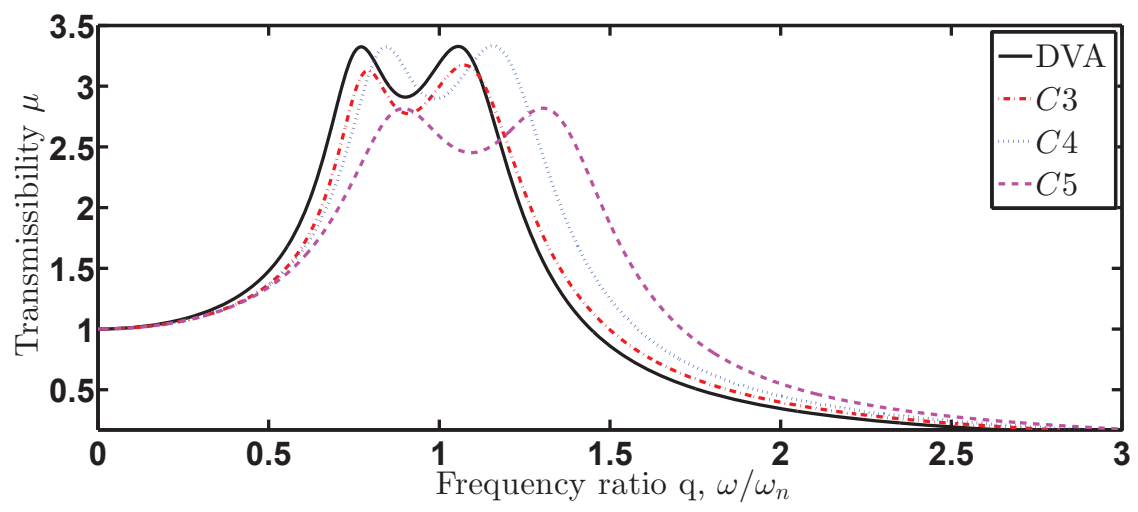

Figure 12: Comparison between traditional DVA and inerter-based isolators when $\delta=0.2$.

\subsection{Comparison between the traditional DVA and the inerter-based isolators}

Now, all the optimal parameters for these inerter-based isolators in $H_{\infty}$ optimisation have been derived. In this section, the performance of the inerter-based isolators will be compared with the traditional DVA as shown in Fig. 11. For the traditional DVA,

$$
\mu=\sqrt{\frac{A \zeta^{2}+B}{C \zeta^{2}+D}},
$$

where $A=4 \lambda^{2} q^{2}, B=\left(1-\delta \lambda q^{2}\right)^{2}, C=4 \lambda^{2}\left(1-(1+\delta) q^{2}\right)^{2} q^{2} \zeta^{2}+\left(1-(1+\delta+\delta \lambda) q^{2}+\delta \lambda q^{4}\right)^{2}$, and the mass ratio $\delta$ and the stiffness ratio $\lambda$ are defined as $\delta=\frac{m_{a}}{m}$ and $\lambda=\frac{k}{k_{a}}$, respectively.

It is well known that the optimal parameters for the traditional DVA $[25,26,27]$ are

$$
\lambda_{\text {opt }}=\frac{(\delta+1)^{2}}{\delta}, \zeta_{\text {opt }}=\frac{\delta}{1+\delta} \sqrt{\frac{3 \delta}{8(1+\delta)}} .
$$

Fig. 12 shows the comparison between the traditional DVA and the inerter-based isolators when the inertance-to-mass ratio (or mass ratio for traditional DVA) $\delta=0.2$, where it is clearly shown that in terms of the same $\delta$, the configuration $C 4$ provides comparable performance compared with the traditional DVA; whereas both $C 3$ and $C 5$ perform better than the traditional DVA. Such an observation is confirmed by Fig. 13, where the comparison of the maximal $\mu$ with respect to different $\delta$ is shown. The comparison of the optimal stiffness ratio $\lambda$ and damping ratio $\zeta$ with respect to different $\delta$ is shown in Fig. 14.

Note that the fundamental difference between the traditional DVA and the inerter-based isolators is that the inertance-to-mass ratio of the inerter-based isolators can easily be larger than the mass ratio of the traditional DVA, as large inertance can easily be obtained without increasing the physical mass of the whole system. For example, the inertance of a rack-pinion inerter or a ball-screw inerter can be significantly magnified by enlarging the gear ratios $[1,2]$. However, the mass ratio $\delta$ for the traditional DVA is practically less than $0.25[26,28]$. From this point of view, the performance of the inerter-based isolators can be further improved compared with the traditional DVA, and the inerter-based isolators are potentially more attractive than the traditional DVA. 


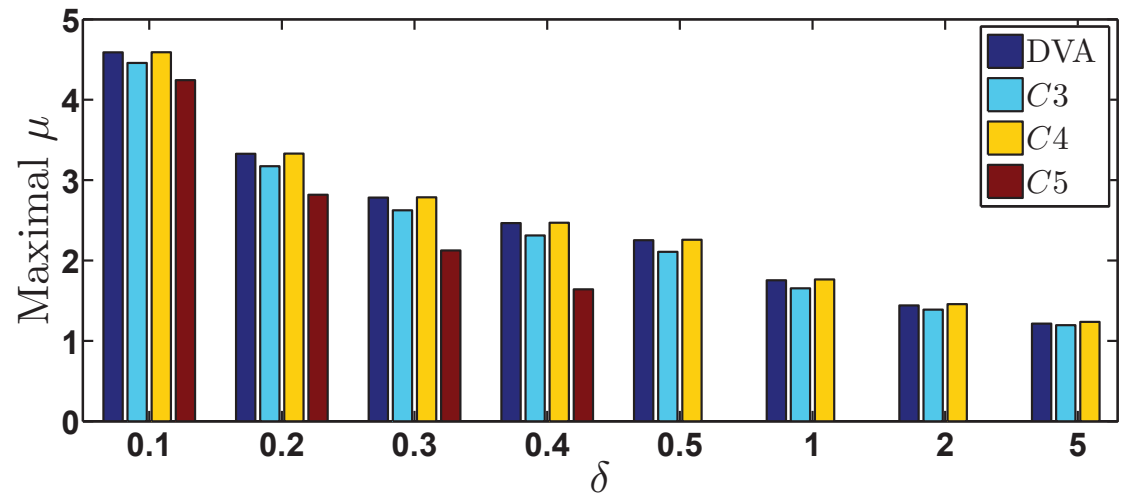

Figure 13: Comparison of the maximal $\mu$ in $H_{\infty}$ optimisation.

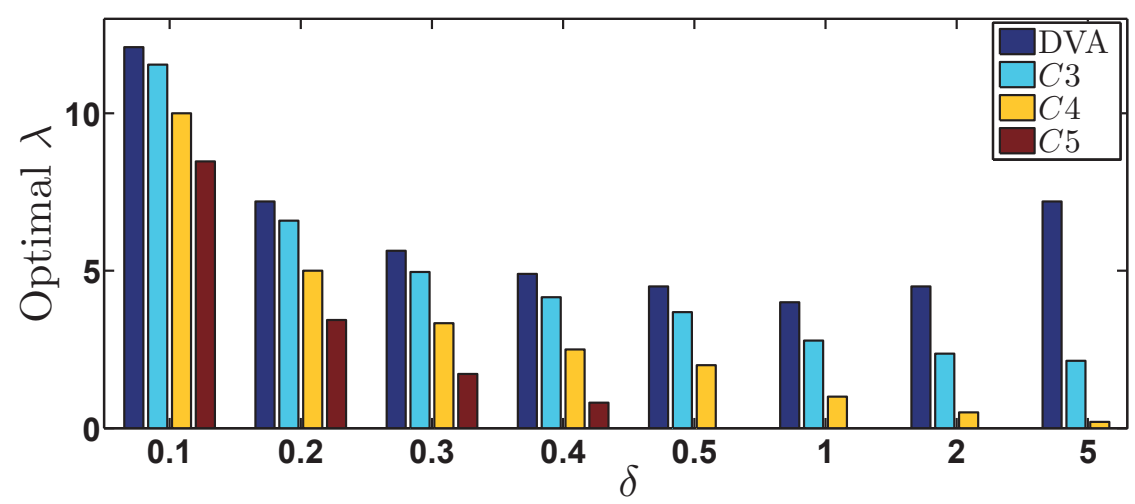

(a)

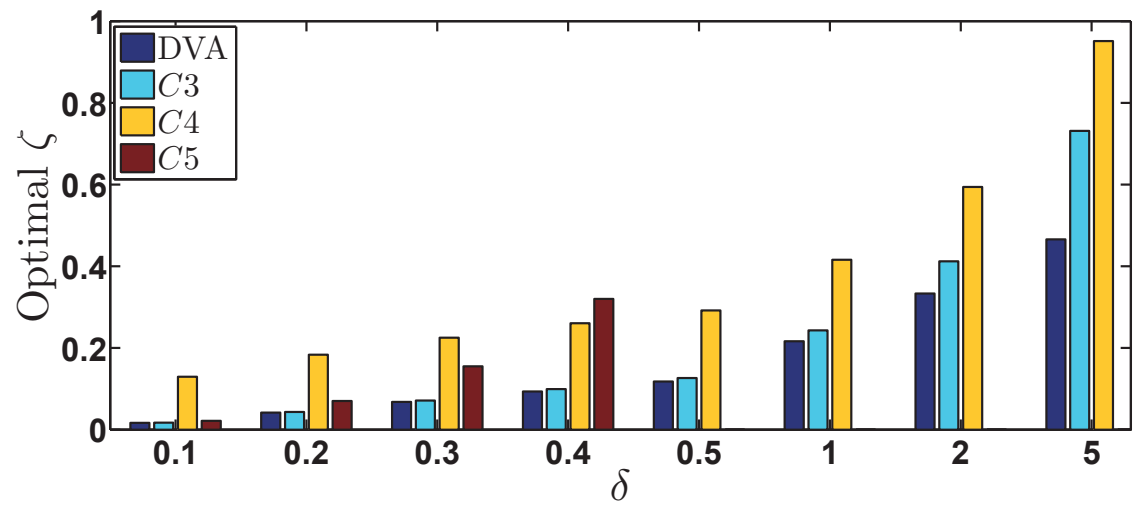

(b)

Figure 14: Comparison of the optimal parameters in $H_{\infty}$ optimization. (a) Optimal stiffness ratio $\lambda$; (b) optimal damping ratio $\zeta$. 


\section{5. $\mathrm{H}_{2}$ optimisation for inerter-based isolators}

$\mathrm{H}_{2}$ optimisation aims to minimise the total vibration energy or the mean square motion of the object mass when white noise excitation is enforced [29]. In the case of random excitation such as wind loading instead of harmonic excitation, the $\mathrm{H}_{2}$ optimisation would be more practical than the $H_{\infty}$ optimisation. In this section, the analytical solutions for the inerter-based isolators in $H_{2}$ optimisation will be derived and compared with the traditional DVA.

The performance measure to be minimised in $H_{2}$ optimisation is defined as follows [29, 27]:

$$
I=\frac{E\left[x_{1}^{2}\right]}{2 \pi S_{0} \omega_{n}},
$$

where $S_{0}$ is the uniform power spectrum density function. Denoting $\mu=|H(\mathrm{j} q)|$, the mean square value of $x_{1}$ of the object mass $m$ can be calculated as

$$
E\left[x_{1}^{2}\right]=S_{0} \int_{-\infty}^{\infty}|H(\mathrm{j} q)|^{2} \mathrm{~d} \omega=S_{0} \omega_{n} \int_{-\infty}^{\infty}|H(\mathrm{j} q)|^{2} \mathrm{~d} q .
$$

Substituting (31) into (30), one obtains

$$
I=\frac{1}{2 \pi} \int_{-\infty}^{\infty}|H(\mathrm{j} q)|^{2} \mathrm{~d} q,
$$

which is exactly the definition of the $H_{2}$ norm of the transfer function $\hat{H}(s)$ by replacing $\mathrm{j} q$ in $H(\mathrm{j} q)$ with the Laplace variable $s$.

Therefore, the $\mathrm{H}_{2}$ performance measure is rewritten as

$$
I=\|\hat{H}(s)\|_{2}^{2} .
$$

In what follows, an analytical approach to calculating the $H_{2}$ norm of the transfer function $\hat{H}(s)$ will be presented according to [32, Chapter 2.6], which has been used to derive analytical solutions for vehicle suspensions in $[6,7]$.

For a stable transfer function $\hat{H}(s)$, its $H_{2}$ norm can be calculated as [32, Chapter 2.6]

$$
\|\hat{H}(s)\|_{2}^{2}=\left\|C(s I-A)^{-1} B\right\|_{2}^{2}=C L C^{T},
$$

where $A, B, C$ are the minimal state-space realization $\hat{H}(s)=C(s I-A)^{-1} B$ and $L$ is the unique solution of the Lyapunov equation

$$
A L+L A^{T}+B B^{T}=0 .
$$

We can write $\hat{H}(s)$

$$
\hat{H}(s)=\frac{b_{n-1} s^{n-1}+\ldots+b_{1} s+b_{0}}{s^{n}+a_{n-1} s^{n-1}+\ldots+a_{1} s+a_{0}}
$$

in its controllable canonical form below

$$
\dot{x}=A x+B u, y=C x,
$$


where

$$
A=\left[\begin{array}{ccccc}
0 & 1 & 0 & \ldots & 0 \\
0 & 0 & 1 & \ldots & 0 \\
\vdots & \vdots & \vdots & \vdots & \vdots \\
0 & 0 & 0 & \ldots & 1 \\
-a_{0} & -a_{1} & -a_{2} & \ldots & -a_{n-1}
\end{array}\right], B=\left[\begin{array}{c}
0 \\
0 \\
\vdots \\
0 \\
1
\end{array}\right], C=\left[b_{0}, b_{1}, b_{2} \ldots b_{n-1}\right]
$$

Note that the analytical solution for the configuration $C 1$ cannot be derived by using the above method, as the $\hat{H}(s)$ for $C 1$ is not strictly proper. Actually, the $H_{2}$ norm of $\hat{H}(s)$ for $C 1$ is infinity which can be obtained by observing Fig. 4: the area under the frequency response curve of $C 1$ which represents the $H_{2}$ norm of the transfer function is infinity.

The procedure to derive the optimal parameters for $C 2, C 3, C 4$ and $C 5$ can be summarised as:

\section{Procedure 3.}

1. analytically calculate the $\mathrm{H}_{2}$ performance measure $I$ using the method discussed above. Denote the performance measure as $I=F(\lambda) \zeta+\frac{G(\lambda)}{\zeta}$, where $F(\lambda)$ and $G(\lambda)$ are functions of $\lambda$ with $F(\lambda)>0, G(\lambda)>0$;

2. obtain the equations of optimal $\zeta$ and $I$ as $\zeta_{\text {opt }}=\sqrt{\frac{F(\lambda)}{G(\lambda)}}$ and $I_{\text {opt }}=2 \sqrt{F(\lambda) G(\lambda)}$, respectively;

3. obtain the optimal $\lambda$ as the one minimising $F(\lambda) G(\lambda)$, denoted as $\lambda_{\text {opt }}$;

4. obtain the optimal $\zeta$ and $I$ by substituting $\lambda_{\text {opt }}$ into the equations obtained in Step 2, respectively.

Note that in Step 1 of Procedure 3, it includes the case that $F(\lambda)$ and $G(\lambda)$ are constants with respect to $\lambda$. Following Procedure 3, the optimal parameters for $C 2, C 3, C 4$, and $C 5$ in the $H_{2}$ optimisation will be derived subsequently.

Proposition 5. For the configuration $\mathrm{C} 2$, the $\mathrm{H}_{2}$ performance measure in (32) is

$$
I_{c 2}=\frac{1-\delta+\delta^{2}}{\delta^{2}} \zeta+\frac{1}{4 \zeta}
$$

For a given $\delta$, the optimal $\zeta$ is

$$
\zeta_{o p t}=\frac{\delta}{2 \sqrt{1-\delta+\delta^{2}}}
$$

After substituting $\zeta_{\text {opt }}$ into (35), the optimal $I_{c 2}$ is

$$
I_{c 2, o p t}=\frac{\sqrt{1-\delta+\delta^{2}}}{2 \delta} \text {. }
$$

Proof. Equation (35) can be obtained by direct calculation, and then the optimal $\zeta$ and $I_{c 2, \text { opt }}$ can be obtained subsequently. 
Proposition 6. For the configuration $\mathrm{C} 3$, the $\mathrm{H}_{2}$ performance measure in (32) is

$$
I_{c 3}=\frac{1-\delta+\delta^{2}}{\delta^{2}} \zeta+\frac{1-2 \delta \lambda+\delta^{2} \lambda^{2}+\delta^{2} \lambda}{4 \lambda^{2} \delta^{2} \zeta} .
$$

For a given $\delta$, the optimal $\lambda$ can be obtained as

$$
\lambda_{\text {opt }}= \begin{cases}\frac{2}{\delta(2-\delta)}, & \delta<2 \\ \infty, & \delta \geq 2\end{cases}
$$

Note that in the case of $\delta \geq 2, C 3$ reduces to $C 2$. For a given $\delta$ and $\lambda$, the optimal $\zeta$ can be obtained as

$$
\zeta_{o p t}=\frac{1}{2 \lambda} \sqrt{\frac{1-2 \delta \lambda+\delta^{2} \lambda}{1-\delta+\delta^{2}}} .
$$

Then, the optimal $I_{c 3}$ can be obtained by substituting $\zeta_{\text {opt }}$ and $\lambda_{\text {opt }}$ into (36).

Proof. Equation (36) can be obtained by direct calculation. The optimal $\lambda$ can be obtained by checking the second part in (36). Since both parts in (36) are positive, the optimal $\zeta$ can be obtained subsequently.

Proposition 7. For the configuration $\mathrm{C} 4$, the $\mathrm{H}_{2}$ performance measure in (32) is

$$
I_{c 4}=\frac{1-2 \delta \lambda+\delta^{2} \lambda^{2}+2 \delta^{2} \lambda-\delta+\delta^{2}}{\delta^{2}} \zeta+\frac{1}{4 \zeta} .
$$

For a given $\delta$, the optimal $\lambda$ can be obtained as

$$
\lambda_{\text {opt }}= \begin{cases}\frac{1-\delta}{\delta}, & \delta<1 \\ 0, & \delta \geq 1\end{cases}
$$

Note that in the case of $\delta \geq 1, C 4$ reduces to $C 2$. For a given $\delta$ and $\lambda$, the optimal $\zeta$ can be obtained as

$$
\zeta_{\text {opt }}=\frac{1}{2} \sqrt{\frac{\delta^{2}}{1-2 \delta \lambda+\delta^{2} \lambda^{2}+2 \delta^{2} \lambda-\delta+\delta^{2}}} .
$$

Then, the optimal $I_{c 4}$ can be obtained by substituting $\zeta_{\text {opt }}$ and $\lambda_{\text {opt }}$ into (37).

Proof. The proof is omitted as it is similar to that of Proposition 6.

Proposition 8. For the configuration $\mathrm{C5}$, the $\mathrm{H}_{2}$ performance measure in (32) is

$$
I_{c 5}=(\lambda+1)^{2} \zeta+\frac{\delta^{3} \lambda^{3}+\delta(3 \delta-2) \lambda^{2}+\left(1-2 \delta+3 \delta^{3}\right) \lambda+\delta^{2}}{4 \lambda \zeta} .
$$

For a given $\delta$ and $\lambda$, the optimal $\zeta$ and $I_{c 5}$ can be obtained as

$$
\begin{aligned}
\zeta_{o p t} & =\frac{1}{2(1+\lambda)} \sqrt{\frac{\delta^{3} \lambda^{3}+\delta(3 \delta-2) \lambda^{2}+\left(1-2 \delta+3 \delta^{3}\right) \lambda+\delta^{2}}{\lambda}}, \\
I_{c 5, o p t} & =(\lambda+1) \sqrt{\frac{\delta^{3} \lambda^{3}+\delta(3 \delta-2) \lambda^{2}+\left(1-2 \delta+3 \delta^{3}\right) \lambda+\delta^{2}}{\lambda}} .
\end{aligned}
$$


Let $\mathcal{Q}$ be the set of real, positive solutions $\lambda$ of the quartic equation

$$
4 \delta^{2} \lambda^{4}+(11 \delta-6) \delta \lambda^{3}+\left(2-6 \delta+9 \delta^{2}\right) \lambda^{2}+\delta^{2} \lambda-\delta^{2}=0 .
$$

The optimal $\lambda$ is chosen from the elements of $\mathcal{Q}$ as well as 0 that makes $I_{c 5, \text { opt }}$ minimum. If the optimal $\lambda$ is 0 , configuration $C 5$ reduces to $C 1$.

Proof. Equation (38) can be obtained by direct calculation. Since both parts in (38) are positive, the optimal $\zeta$ and $I_{c 5}$ can be obtained as in (39) and (40) respectively in a straightforward manner. In terms of (40), by making the derivative of $I_{c 5, \text { opt }}$ with respect to $\lambda$ zero, the quartic equation (41) can be obtained, and then the optimal $\lambda$ can be selected from the real, positive solutions of the quartic equation as well as $\infty$.

\subsection{Comparison between the traditional DVA and the inerter-based isolators}

Now, all the optimal parameters for the inerter-based isolators in $H_{2}$ optimisation have been derived. In this section, the performance of these inerter-based isolators will be compared with the traditional DVA as shown in Fig. 11.

For the traditional DVA shown in Fig. 11, the $H_{2}$ performance measure can be derived as

$$
I_{D V A}=\frac{1+\delta}{\delta} \zeta+\frac{(\delta+1)^{2}-\delta(\delta+2) \lambda+\delta^{2} \lambda^{2}}{4 \lambda^{2} \delta^{2} \zeta},
$$

where the mass ratio $\delta$ and the stiffness ratio $\lambda$ are defined as $\delta=m_{a} / m$ and $\lambda=k / k_{a}$.

Similar to the inerter-based isolators, the optimal parameters can be obtained as:

$$
\lambda_{\text {opt }}=\frac{2(\delta+1)^{2}}{\delta(\delta+2)},
$$

$$
\zeta_{\text {opt }}=4 \sqrt{\frac{\delta^{3}(3 \delta+4)}{(\delta+1)^{3}}}
$$

$$
I_{D V A, o p t}=\frac{1}{2} \sqrt{\frac{3 \delta+4}{\delta(\delta+1)}} .
$$

Fig. 15, Fig. 16 and Fig. 17 show the comparison between the traditional DVA and the inerter-based isolators in $\mathrm{H}_{2}$ optimisation. As shown in Fig. 15, for the same $\delta$, the inerterbased isolator $C 5$ and $C 3$ perform better than the traditional DVA when $\delta$ less than 0.44 and 1.2, respectively, and the configuration $C 3$ performs slightly worse than the traditional DVA. As shown in Fig. 15, when $\delta<0.44$, the configuration $C 5$ performs best among all the inerter-based isolators. From Fig. 16, it is shown that the damping ratios $\zeta$ of the inerter-based isolators are normally smaller than the traditional DVA. The detailed values of the parameters are given in Table 2, where it is shown that when $\delta=0.2$, the inerterbased isolator $C 3$ and $C 5$ can provide $8.75 \%$ and $49.06 \%$ improvement compared with the traditional DVA.

Similar to the $H_{\infty}$ optimisation, the fundamental difference between the traditional DVA and the inerter-based isolators is that relatively large value of inertance can easily be achieved without increasing the physical mass of the isolation system $[1,2]$; whereas the attached mass 


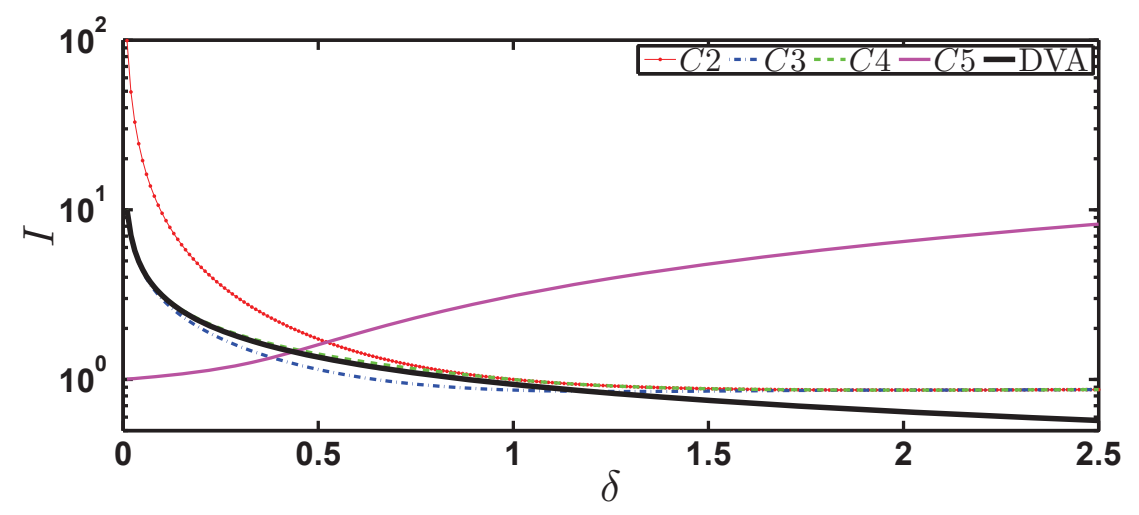

Figure 15: Comparison between traditional DVA and inerter-based isolators in $H_{2}$ optimisation.

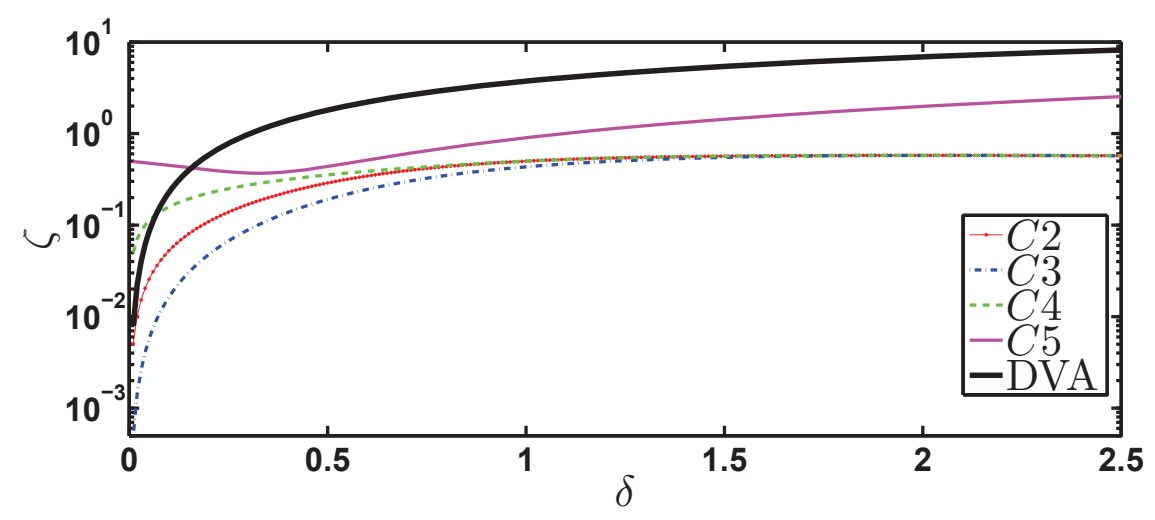

Figure 16: Optimal damping ratio $\zeta$ in $H_{2}$ optimisation.

$m_{a}$ is normally quite small and the typical mass ratio $\delta$ for the traditional DVA is less than $0.25[26,28]$. In this sense, the performance of the inerter-based isolators can be further improved by increasing the inertance-to-mass ratio $\delta$ even $\delta>0.25$, which is a potential advantage of the inerter-based isolators compared with the traditional DVA.

\section{Conclusions}

In this paper, the performance of inerter-based isolators has been investigated by applying five configurations with inerter in a "uni-axial" isolation system. In the first part of this paper, the frequency responses of the inerter in parallel connection and the one in series connection are analysed. It has been analytically demonstrated that both the parallel-connected inerter and the series-connected one can effectively lower the invariant points, and the isolation for high frequencies can be weakened by using inerter. In the second part of this paper, both $H_{\infty}$ and $H_{2}$ performances have been considered for the proposed inerter-based isolators. The fixed-point theory and the analytical method in calculating $H_{2}$ norm are employed to analytically derive the optimal parameters in $H_{\infty}$ and $H_{2}$ optimisation, respectively. The performances of the inerter-based isolators have also been compared with the traditional DVA to show the benefits of the inerter-based isolators. On one hand, it has been shown that for the same mass ratio or inertance-to-mass ratio, two inerter-based isolators perform better than the traditional DVA. On the other hand, two unique properties make the inerter- 
Table 2: Comparison of optimal parameters in $H_{2}$ optimisation.

(a) $\mathrm{H}_{2}$ performance measure $I$

\begin{tabular}{llllll}
\hline$\delta$ & DVA & $C 2$ & $C 3$ & $C 4$ & $C 5$ \\
\hline 0.1 & 3.1261 & 9.5394 & 2.9787 & 3.1623 & 1.0479 \\
0.2 & 2.1890 & 4.5826 & 1.9975 & 2.2361 & 1.1152 \\
0.3 & 1.7723 & 2.9627 & 1.5607 & 1.8257 & 1.2184 \\
0.4 & 1.5236 & 2.1794 & 1.3077 & 1.5811 & 1.3798 \\
0.5 & 1.3540 & 1.7321 & 1.1456 & 1.4142 & 1.6015 \\
1 & 0.9354 & 1.0000 & 0.8660 & 1.0000 & 3.1087 \\
2 & 0.6455 & 0.8660 & 0.8660 & 0.8660 & 6.5065 \\
5 & 0.3979 & 0.9165 & 0.9165 & 0.9165 & 16.9393 \\
\hline
\end{tabular}

(b) optimal stiffness ratio $\lambda$

\begin{tabular}{lllll}
\hline$\delta$ & DVA & $C 3$ & $C 4$ & $C 5$ \\
\hline 0.1 & 11.5238 & 10.5263 & 9.0000 & 0.0796 \\
0.2 & 6.5455 & 5.5556 & 4.0000 & 0.1787 \\
0.3 & 4.8986 & 3.9216 & 2.3333 & 0.2824 \\
0.4 & 4.0833 & 3.1250 & 1.5000 & 0.3426 \\
0.5 & 3.6000 & 2.6667 & 1.0000 & 0.3542 \\
1 & 2.6667 & 2.0000 & 0 & 0.3139 \\
2 & 2.2500 & $\infty$ & 0 & 0.2815 \\
5 & 2.0571 & $\infty$ & 0 & 0.2623 \\
\hline
\end{tabular}

(c) optimal damping ratio $\zeta$

\begin{tabular}{llllll}
\hline$\delta$ & DVA & $C 2$ & $C 3$ & $C 4$ & $C 5$ \\
\hline 0.1 & 0.2274 & 0.0524 & 0.0164 & 0.1581 & 0.4495 \\
0.2 & 0.5837 & 0.1091 & 0.0476 & 0.2236 & 0.4014 \\
0.3 & 0.9816 & 0.1688 & 0.0889 & 0.2739 & 0.3704 \\
0.4 & 1.3930 & 0.2294 & 0.1376 & 0.3162 & 0.3827 \\
0.5 & 1.8053 & 0.2887 & 0.1909 & 0.3536 & 0.4367 \\
1 & 3.7417 & 0.5000 & 0.4330 & 0.5000 & 0.9004 \\
2 & 6.8853 & 0.5774 & 0.5774 & 0.5774 & 1.9810 \\
5 & 13.2637 & 0.5455 & 0.5455 & 0.5455 & 5.3157 \\
\hline
\end{tabular}




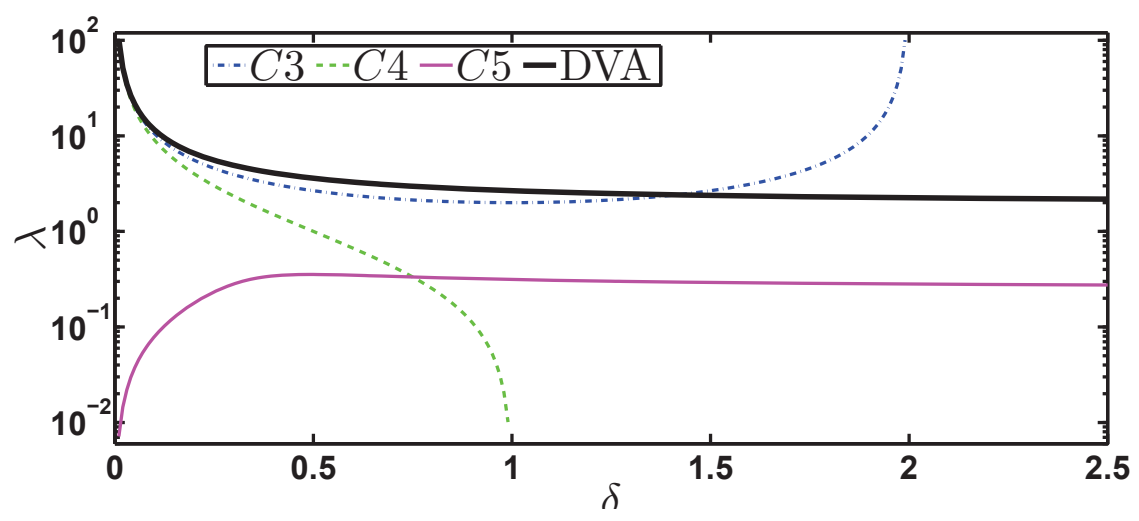

Figure 17: Optimal stiffness ratio $\lambda$ in $H_{2}$ optimisation.

based isolators potentially more attractive than the traditional DVA: first, a large inertance can easily be obtained for inerter without increasing the physical mass of the whole system; second, the inerter is a built-in element and there is no need to mount an additional mass to the object to be isolated.

In practical applications of the inerter-based isolators, the large transmission ratios employed in the physical embodiments of inerter will amplify the internal friction of the rotating device with a gain that is equal to the square of the transmission ratio. This could lead to an amount of damping at a system level larger than the optimal one, which may render the proposed inerter-based isolators far from an ideal design. More research work needs to be carried to find low-friction designs to be used with high amplification ratio.

\section{Appendix A. Proof of Proposition 1}

Observing Fig. 5, it is shown that the curve horizontally passing through $P$ indicates the optimal damping. This optimal damping can be obtained by solving the following equation

$$
\left.\frac{\partial \mu^{2}}{\partial q^{2}}\right|_{q=q_{P}}=0
$$

Denote $\mu=\sqrt{\frac{n}{m}}$, where $n=\delta^{2} q^{2}+4\left(1-\delta q^{2}\right)^{2} \zeta^{2}, m=\delta^{2}\left(1-q^{2}\right)^{2} q^{2}+4\left(1-(1+\delta) q^{2}\right)^{2} \zeta^{2}$. Equation (A.1) can be written in another form as

$$
n^{\prime} m-m^{\prime} n=0,
$$

where $n^{\prime}=\partial n / \partial q^{2}$, and $m^{\prime}=\partial m / \partial q^{2}$. For the invariant point $P$,

$$
\frac{n}{m}=\frac{1}{\left(1-q^{2}\right)^{2}}=\frac{\left(1-\delta q^{2}\right)^{2}}{\left(1-(1+\delta) q^{2}\right)^{2}},
$$

therefore,

$$
\left(1-q^{2}\right)^{2} n^{\prime}-m^{\prime}=0
$$

Since

$$
n^{\prime}=-8\left(1-\delta q^{2}\right) \delta \zeta^{2}+\delta^{2}
$$




$$
m^{\prime}=-8\left(1-(1+\delta) q^{2}\right)(\delta+1) \zeta^{2}+\delta^{2}\left(1-q^{2}\right)\left(1-3 q^{2}\right),
$$

413

\section{Appendix B. Proof of Proposition 2}

Denote

$$
\begin{gathered}
A=4 \lambda^{2}\left(1-\delta q^{2}\right)^{2} q^{2}, B=\left(1-\delta(1+\lambda) q^{2}\right)^{2}, \\
C=4 \lambda^{2}\left(1-(1+\delta) q^{2}\right)^{2} q^{2}, D=\left(1-(\delta+1+\delta \lambda) q^{2}+\delta \lambda q^{4}\right)^{2} .
\end{gathered}
$$

Then, $\mu$ in (14) can be rewritten as

$$
\mu=\sqrt{\frac{A \zeta^{2}+B}{C \zeta^{2}+D}} .
$$

To find the invariant points which are independent of damping, it requires

$$
\frac{A}{C}=\frac{B}{D}
$$

that is,

$$
\frac{1-\delta q^{2}}{1-(1+\delta) q^{2}}= \pm \frac{1-\delta(1+\lambda) q^{2}}{1-(\delta+1+\delta \lambda) q^{2}+\delta \lambda q^{4}}
$$

With the plus sign, after cross multiplication, one obtains $\delta^{2} \lambda q^{6}=0$, which leads to the trivial solution $q=0$. With the minus sign, after simple calculation, one obtains

$$
\delta^{2} \lambda q^{6}-2 \delta(\lambda+\delta+1+\delta \lambda) q^{4}+2(2 \delta+1+\delta \lambda) q^{2}-2=0,
$$

which is a cubic form in $q^{2}$. Therefore, there are three invariant points for the configuration $C 3$.

Denoting these three invariant points as $P, Q$ and $R\left(q_{P}<q_{Q}<q_{R}\right)$, separately, one obtains

$$
\begin{aligned}
q_{P}^{2}+q_{Q}^{2}+q_{R}^{2} & =\frac{2}{\delta \lambda}(\lambda+\delta+1+\lambda \delta), \\
q_{P}^{2} q_{Q}^{2} q_{R}^{2} & =\frac{2}{\delta^{2} \lambda} \\
q_{P}^{2} q_{Q}^{2}+q_{P}^{2} q_{R}^{2}+q_{Q}^{2} q_{R}^{2} & =\frac{2}{\delta^{2} \lambda}(2 \delta+1+\delta \lambda) .
\end{aligned}
$$

Since at points $P$ and $Q$, the values of $\mu$ are independent of $\zeta$, then in the case of $\zeta=\infty$, one obtains

$$
\left|\frac{1-\delta q_{P}^{2}}{1-(1+\delta) q_{P}^{2}}\right|=\left|\frac{1-\delta q_{Q}^{2}}{1-(1+\delta) q_{Q}^{2}}\right| .
$$


It can be checked that

$$
\frac{1-\delta q_{P}^{2}}{1-(1+\delta) q_{P}^{2}}>0, \frac{1-\delta q_{Q}^{2}}{1-(1+\delta) q_{Q}^{2}}<0
$$

Then, one obtains

$$
\frac{1-\delta q_{P}^{2}}{1-(1+\delta) q_{P}^{2}}=-\frac{1-\delta q_{Q}^{2}}{1-(1+\delta) q_{Q}^{2}}
$$

After cross multiplication and simplification, one obtains

$$
2 \delta(1+\delta) q_{P}^{2} q_{Q}^{2}-\left(q_{P}^{2}+q_{Q}^{2}\right)(1+2 \delta)+2=0 .
$$

Substituting (B.4) and (B.5) into (B.6), one can obtains a quadratic equation with respect to $q_{R}^{2}$ as

$$
\delta \lambda(1+2 \delta) q_{R}^{4}-2\left(\lambda+2 \delta \lambda+3 \delta+2 \delta^{2}+1+2 \lambda \delta^{2}\right) q_{R}^{2}+4(1+\delta)=0 .
$$

Note that $q_{R}$ is the same solution as both (B.2) and (B.7) for the same $\delta$ and $\lambda$. Solving $\lambda$ from (B.2) and (B.7), separately, one obtains

$$
\begin{aligned}
\lambda & =\frac{2\left(q_{R}^{4} \delta(1+\delta)-(1+2 \delta) q_{R}^{2}+1\right)}{\delta q_{R}^{2}\left(q_{R}^{4} \delta-2(\delta+1) q_{R}^{2}+2\right)}, \\
\lambda & =\frac{2\left((1+2 \delta)(1+\delta) q_{R}^{2}-2(1+\delta)\right)}{q_{R}^{2}\left(\delta(1+2 \delta) q_{R}^{2}-2\left(1+2 \delta+2 \delta^{2}\right)\right)} .
\end{aligned}
$$

Equating the solutions and simplifying the results, one obtains

$$
\delta q_{R}^{4}-(2+3 \delta) q_{R}^{2}+2=0 .
$$

Then, one obtains $q_{R}^{2}$ as shown in (15).

From (15), it is easy to show that $q_{R}^{2} \geq 3$, which is relatively large compared with the natural frequency. This can explain why only invariant points $P$ and $Q$ are involved in the $H_{\infty}$ tuning of $C 3$.

In this way, the optimal $\lambda$ can be obtained by substituting $q_{R}^{2}$ in (15) into (B.8) or (B.9). After obtaining $\lambda$, all the three invariant points can be obtained by solving

$$
q^{4}-\left(\frac{2}{\delta \lambda}(1+\lambda+\delta+\lambda \delta)-q_{R}^{2}\right) q^{2}+\frac{2}{\delta^{2} \lambda q_{R}^{2}}=0,
$$

which is obtained from (B.4) and (B.5).

The procedure of calculating the optimal damping ratio $\zeta$ is similar to the procedure in Appendix Appendix A, where the optimal $\zeta$ makes the gradients at invariant points $P$ and $Q$ zero. After calculation and simplification, one obtains (18). Taking an average of $\zeta_{P}^{2}$ and $\zeta_{Q}^{2}$, one obtains the optimal $\zeta_{\text {opt }}$ as in (17). 


\section{Appendix C. Proof of Proposition 3}

Denote

$$
\begin{gathered}
A=4\left(1-\delta(1+\lambda) q^{2}\right)^{2}, B=\delta^{2} q^{2}, \\
C=4\left(1-(1+\delta+\delta \lambda) q^{2}+\delta \lambda q^{4}\right)^{2}, D=\delta^{2}\left(1-q^{2}\right)^{2} q^{2},
\end{gathered}
$$

and $\mu$ in $(20)$ can be rewritten as

$$
\mu=\sqrt{\frac{A \zeta^{2}+B}{C \zeta^{2}+D}}
$$

To find the invariant points which are independent of damping, it requires

$$
\frac{A}{C}=\frac{B}{D}
$$

that is,

$$
\frac{1-\delta(1+\lambda) q^{2}}{1-(1+\delta+\delta \lambda) q^{2}+\delta \lambda q^{4}}= \pm \frac{1}{1-q^{2}}
$$

Again, with the plus sign, one obtains the trivial solution zero, and with the minus sign, one obtains

$$
\delta(1+2 \lambda) q^{4}-2(1+\delta+\delta \lambda) q^{2}+2=0 .
$$

Then, one obtains the two invariant points $P$ and $Q\left(q_{P}<q_{Q}\right)$ as

$$
q_{P, Q}^{2}=\frac{1+\delta+\delta \lambda \pm \sqrt{(1+\delta+\delta \lambda)^{2}-2 \delta(1+2 \lambda)}}{\delta(1+2 \lambda)} .
$$

Letting the ordinates at invariant points $P$ and $Q$ equal, one has

$$
\left|\frac{1}{1-q_{P}^{2}}\right|=\left|\frac{1}{1-q_{Q}^{2}}\right| \text {. }
$$

${ }_{457}$ It can be checked that $\frac{1}{1-q_{P}^{2}}>0$ and $\frac{1}{1-q_{Q}^{2}}<0$. Then, one obtains

$$
\frac{1}{1-q_{P}^{2}}=-\frac{1}{1-q_{Q}^{2}} .
$$

After cross multiplication and simplification, one has

$$
q_{P}^{2}+q_{Q}^{2}=2
$$

Considering (C.2), one obtains

$$
\frac{2(1+\delta+\delta \lambda)}{\delta(1+2 \lambda)}=2
$$

which leads to (21).

Similar to the method in Appendix Appendix A, the optimal $\zeta$ can be obtained by making $\mu$ have zero gradients at invariant points $P$ and $Q$. After calculation and simplification, one obtains

$$
\zeta_{P, Q}^{2}=\frac{q_{P, Q}^{2} \delta^{2}}{4\left(1-\delta(1+\lambda) q_{P, Q}^{2}\right)\left(1+2 \delta+2 \delta \lambda-\delta(1+3 \lambda) q_{P, Q}^{2}\right)} .
$$

After substituting (C.3) and (21), one obtains (23) and (24).

Taking an average of $\zeta_{p}^{2}$ and $\zeta_{Q}^{2}$, one obtains the optimal $\zeta_{\text {opt }}$ as in (22). 


\section{Appendix D. Proof of Proposition 4}

Denote

$$
\begin{gathered}
A=4(\lambda+1)^{2} q^{2}, B=\left(1-\delta(1+\lambda) q^{2}\right)^{2}, \\
C=4\left(\lambda+1-\lambda q^{2}\right)^{2} q^{2}, D=\left(1-(1+\delta+\delta \lambda) q^{2}+\lambda \delta q^{4}\right)^{2} .
\end{gathered}
$$

Then, $\mu$ in $(25)$ can be rewritten as

$$
\mu=\sqrt{\frac{A \zeta^{2}+B}{C \zeta^{2}+D}} .
$$

To find the invariant points which are independent of damping, it requires

$$
\frac{A}{C}=\frac{B}{D}
$$

that is,

$$
\frac{\lambda+1}{\lambda+1-\lambda q^{2}}= \pm \frac{1-\delta(1+\lambda) q^{2}}{1-(1+\delta+\delta \lambda) q^{2}+\delta \lambda q^{4}}
$$

Similarly, with plus sign, one obtains the trivial solution zero, and with minus sign, one obtains

$$
2 \delta \lambda(\lambda+1) q^{4}-\left(1+2 \lambda+2 \delta(1+\lambda)^{2}\right) q^{2}+2(\lambda+1)=0 .
$$

44 Thus, one obtains the two invariant points $P$ and $Q\left(q_{P}<q_{Q}\right)$ as in (29).

Letting the ordinates at invariant points $P$ and $Q$ equal, one has

$$
\left|\frac{\lambda+1}{\lambda+1-\lambda q_{P}^{2}}\right|=\left|\frac{\lambda+1}{\lambda+1-\lambda q_{Q}^{2}}\right| \text {. }
$$

It can be checked that $\frac{\lambda+1}{\lambda+1-\lambda q_{P}^{2}}>0$ and $\frac{\lambda+1}{\lambda+1-\lambda q_{Q}^{2}}<0$. Then, one obtains

$$
\frac{\lambda+1}{\lambda+1-\lambda q_{P}^{2}}=-\frac{\lambda+1}{\lambda+1-\lambda q_{Q}^{2}} .
$$

477 After cross multiplication and simplification, one has

$$
q_{P}^{2}+q_{Q}^{2}=\frac{2(\lambda+1)}{\lambda}
$$

48 Comparing with (D.2), one obtains

$$
\frac{1+2 \lambda+2 \delta(1+\lambda)^{2}}{2 \delta \lambda(\lambda+1)}=\frac{2(\lambda+1)}{\lambda},
$$

499 which leads to

$$
2 \delta \lambda^{2}-2(1-2 \delta) \lambda+2 \delta-1=0 .
$$

480 It can be checked that this equation has real solutions if and only if

$$
\delta \leq 1 / 2
$$


Under this condition, the optimal $\lambda$ can be obtained as in (26).

Note that if $\delta=\frac{1}{2}$, from (26), one has $\lambda=0$, or $k=\infty$. In this case $C 5$ reduces to $C 1$. Thus, the more reasonable assumption is $\delta<\frac{1}{2}$ rather than $\delta \leq \frac{1}{2}$.

Similar to the method in Appendix Appendix A, the optimal $\zeta$ can be obtained by making $\mu$ have zero gradients at invariant points $P$ and $Q$. After calculation and simplification, one obtains $\zeta_{P}^{2}$ and $\zeta_{Q}^{2}$ as in $(28)$.

Taking an average of $\zeta_{p}^{2}$ and $\zeta_{Q}^{2}$, one obtains the optimal $\zeta_{\text {opt }}$ as in (27).

\section{Acknowledgements}

The authors are grateful to the Associate Editor and the reviewers for their insightful suggestions.

This research was partially supported by the Research Grants Council, Hong Kong, through the General Research Fund under Grant 17200914, the Innovation and Technology Commission under Grant ITS/178/13, the Natural Science Foundation of China under Grant 61374053, and the National Key Basic Research Scheme of China ("973 Program") under Grant 2012CB720202.

\section{References}

[1] M.C. Smith, Synthesis of mechanical networks: the inerter, IEEE Transactions on Automatic Control 47 (10) (2002) 1648-1662.

[2] M.Z.Q. Chen, C. Papageorgiou, F. Scheibe, F.-C. Wang, M.C. Smith, The missing mechanical circuit element, IEEE Circuits and Systems Magazine 9 (1) (2009) 10-26.

[3] M.C. Smith, F.-C. Wang, Performance benefits in passive vehicle suspensions employing inerters, Vehicle System Dynamics 42 (4) (2004) 235-257.

[4] Y. Hu, C. Li, M.Z.Q. Chen, Optimal control for semi-active suspension with inerter, Proceedings of the 31st Chinese Control Conference, Hefei, China, 2012, pp. 2301-2306.

[5] M.Z.Q. Chen, Y. Hu, C. Li, G. Chen, Performance benefits of using inerter in semiactive suspensions, IEEE Transactions on Control System Technology, in press, DOI:10.1109/TCST.2014.2364954.

[6] Y. Hu, M.Z.Q. Chen, Z. Shu, Passive vehicle suspensions employing inerters with multiple performance requirements, Journal of Sound and Vibration 333 (8) (2014) 2212-2225.

[7] F. Scheibe, M.C. Smith, Analytical solutions for optimal ride comfort and tyre grip for passive vehicle suspensions, Vehile System Dynamics 47 (10) (2009) 1229-1252.

[8] F.-C. Wang, H. A. Chan, Vehicle suspensions with a mechatronic network strut, Vehicle System Dynamics 49 (5) (2011) 811-830.

[9] F.-C. Wang, M.-R. Hsieh, H.-J. Chen, Stability and performance analysis of a full-train system with inerters, Vehicle System Dynamics 50 (4) (2012) 545-571. 
[10] L. Marian, A. Giaralis, Optimal design of a novel tuned mass-damper-inerter (TMDI) passive vibration control configuration for stochastically support-excited structural systems, Probabilistic Engineering Mechanics 38 (2014) 156-164.

[11] P. Brzeski, E. Pavlovskaia, T. Kapitaniak, P. Perlikowski, The application of inerter in tuned mass absorber, International Journal of Non-Linear Mechanics 70 (2015) 20-29.

[12] I.F. Lazar, S.A. Neild, D.J. Wagg, Using an inerter-based device for structural vibration suppression, Earthquake Engineering and Structure Dynamics 43 (8) (2014) 1129-1147.

[13] Y. Hu, M.Z.Q. Chen, Z. Shu, L. Huang, Vibration analysis for isolation system with inerter, Proceedings of the 33rd Chinese Control Conference, Nanjing, China, 2014, pp. 6687-6692.

[14] P.G. Dylejko, I.R. MacGillivray, On the concept of a transmission absorber to suppress internal resonance, Journal of Sound and Vibration 333 (2014) 2719-2734.

[15] M.Z.Q. Chen, A note on PIN polynomials and PRIN rational functions, IEEE Transactions on Circuits and Systems II: Express Briefs 55 (5) (2008) 462-463.

[16] M.Z.Q. Chen, M.C. Smith, A note on tests for positive-real functions, IEEE Transactions on Automatic Control 54 (2) (2009) 390-393.

[17] M.Z.Q. Chen, M.C. Smith, Restricted complexity network realizations for passive mechanical control, IEEE Transactions on Automatic Control 54 (10) (2009) 2290-2301.

[18] K. Wang, M.Z.Q. Chen. Generalized series-parallel RLC synthesis without minimization for biquadratic impedances, IEEE Transactions on Circuits and Systems II: Express Briefs 59 (11) (2012) 766-770.

[19] M.Z.Q. Chen, K. Wang, Y. Zou, J. Lam, Realization of a special class of admittances with one damper and one inerter for mechanical control, IEEE Transactions on Automatic Control 58 (7) (2013) 1841-1846.

[20] M.Z.Q. Chen, K. Wang, Z. Shu, C. Li, Realizations of a special class of admittances with strictly lower complexity than canonical forms, IEEE Transactions on Circuits and Systems-I: Regular Papers 60 (9) (2013) 2465-2473.

[21] M.Z.Q. Chen, K. Wang, M. Yin, C. Li, Z. Zuo, G. Chen, Synthesis of $n$-port resistive networks containing $2 n$ terminals, International Journal of Circuit Theory $\&$ Applications, in press. [DOI: $10.1002 /$ cta.1951]

[22] K. Wang, M.Z.Q. Chen, Y. Hu, Synthesis of biquadratic impedances with at most four passive elements, Journal of the Franklin Institute 351 (3) (2014) 1251-1267.

[23] M.Z.Q. Chen, Y. Hu, L. Huang, G. Chen, Influence of inerter on natural frequencies of vibration systems, Journal of Sound and Vibration 333 (7) (2014) 1874-1887.

[24] J.P. Den Hartog, Mechanical Vibrations, Dover Publications, INC. New York, USA, 1985. 
[25] M.Z. Ren, A variant design of the dynamic vibration absorber, Journal of Sound and Vibration 245 (4) (2001) 762-770.

[26] Y.L. Cheung, W.O. Wong, H-infinity optimization of a variant design of the dynamic vibration absorber - Revisited and new results, Journal of Sound and Vibration 330 (16) (2011) 3901-3912.

[27] T. Asami, T. Wakasono, K. Kameoka, M. Hasegawa, H. Sekiguchi, Optimum design of dynamic absorbers for a system subjected to random excitation, JSME International Journal Series III 34 (2) (1991) 218-226.

[28] D.J. Inman, Engineering Vibration, 3rd Ed., Prentice-Hall, Inc., Upper Saddle River, NJ, 2008.

[29] Y.L. Cheung, W.O. Wong, $\mathrm{H}_{2}$ optimization of a non-traditional dynamic vibration abosorber for vibration control of structures under random force excitation, Journal of Sound and Vibration 330 (6) (2011) 1039-1044.

[30] E.I. Rivin, Passive Vibration Isolation. New York: ASME Press, 2003.

[31] A. Carrella, M.J. Brennan, T.P. Waters, V. Lopes Jr., Force and displacment transimissibility of a nonlinear isolator with high-static-low-dynamic-stiffness, International Journal of Mechanical Sciences 55 (1) (2012) 22-29.

[32] J.C. Doyle, B.A. Francis, A.R. Tannenbaum, Feedback Control Theory, Maxwell Macmillan Int., Oxford, 1992.

[33] O. Nishihara, T. Asami, Closed-form solutions to the exact optimizations of dynamic vibration absorbers (minimizations of the maximum amplitude magnification factors), Journal of Vibration and Acoustics 124 (4) (2002) 576-582. 